\title{
Thermopile Infrared Arrays with ultra wide field of view for person detection and building automation
}

\author{
R. Funk, Dr. J. Schieferdecker, Dr. M. Simon, B. Forg, M. Schnorr, F. Herrmann, C. Schmidt \\ Heimann Sensor GmbH, Maria-Reiche Straße 1, 01109 Dresden \\ info@heimannsensor.com
}

\begin{abstract}
Ultra wide field of view (UWFOV) optics for thermography are suitable for surveillance due to the fact, that a wide area (especially in rooms) can be overviewed with a small number of sensors. Heimann Sensor $\mathrm{GmbH}$ has developed thermopile arrays with $32 \times 32$ pixels (>90×90 deg FOV) and $80 \times 64$ pixels giving an UWFOV optics up to $90^{\circ} \times 120^{\circ}$ observation. Furthermore, it has been accomplished, that objects within a FOV of $150^{\circ}$ can be detected over a rotationally symmetrical image field with a diameter of 91 pixels without significant losses of signal due to vignetting. The UWFOV application has been achieved by utilization of spherical distortion. The optics consists of a two lens aspheric assembly having anti reflective coating. The image space f-number of $F / 0.8$ was a good compromise between high temperature and spatial resolution.
\end{abstract}

Key words: Ultra wide field of view, thermopile array, aspheric lens, surveillance

\section{Simulation and Dimensioning}

The optical requirements refer to a thermopile array from Heimann Sensor with $80 \times 64$ pixels (HTPA80x64d) [1]. Along the 64 pixels the FOV has to reach minimum $90^{\circ} \mathrm{FOV}$. For that it was possible to accomplish the requirements by allowing spherical distortion. In the simulation (Zemax OpticStudio) the usage of aspheric curvatures was permitted. To reach high enough signals, two thin germanium lenses have been chosen during the further optimization steps and the image space $f-$ number was opened to F/0.8.

Simultaneously the spatial resolution was optimized by observing the FFT MTF calculation for 5.56 cycles $/ \mathrm{mm}$. The target of the development was a sharp imaging of a rotationally symmetrical full screen with a diameter of 80 pixels. Therefore few pixels in the corner were neglected concerning signal and resolution due to vignetting and total reflection. Nevertheless during the optimization process it was accomplished that all pixels within a FOV of $150^{\circ}$ were not influenced by signal losses due to vignetting. Heimann Sensor offers further WFOV optics for their TO39 products. [2]

\section{Performance and Application}

In the following we describe the performance of the $80 \times 64$ Array with $90 \times 120$ deg FOV: With the first prototype it could be shown that within a range of $150^{\circ} \mathrm{FOV}$ the maximum loss of signal is $15 \%$ for all pixels. This improves the temperature resolution within a wide FOV range. The measured results presented in Fehler! Verweisquelle konnte nicht gefunden werden., show a high relative illumination within $150^{\circ} \mathrm{FOV}$, while the signal for the corner pixels outside $150^{\circ}$ show a steadily decrease.

In Fig. 3 it is shown, that the simulated FFT MTF of $60 \%$ for 5.56 cycles / $\mathrm{mm}$ is conform to the measured MTF. For example it is possible to resolve a focal object in $5 \mathrm{~m}$ distance with a size of $150 \mathrm{~mm}$. The geometric image analysis in Fig. 3 can visualize based on an $80 \times 80$ grid pattern, how the distortion particularly effects an imaged large scale object. The maximum distortion amounts to $-36.15 \%$.

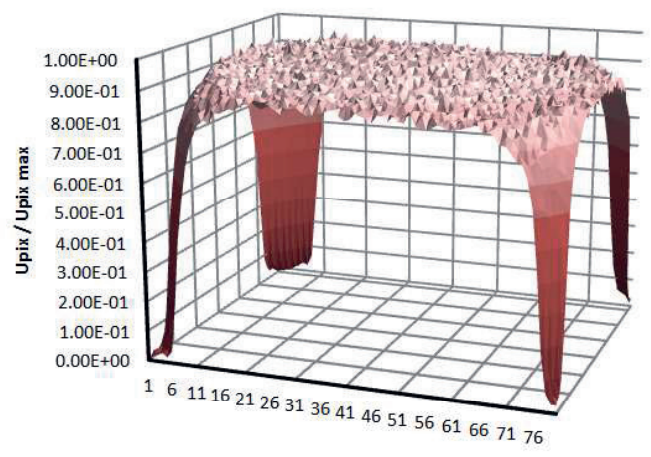

Fig. 1. Measured relative Signal for TPA $80 \times 64 d$ pixel; pixels in the corner are subject to vignetting and total intemal reflection 


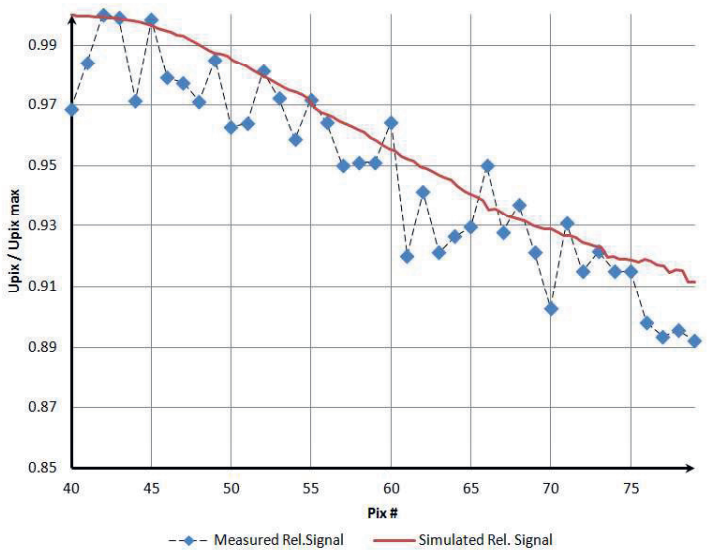

Fig. 1: Comparison of the relative Signal Upix / Upix [max] between simulation and measurement from center to edge pixels along a row
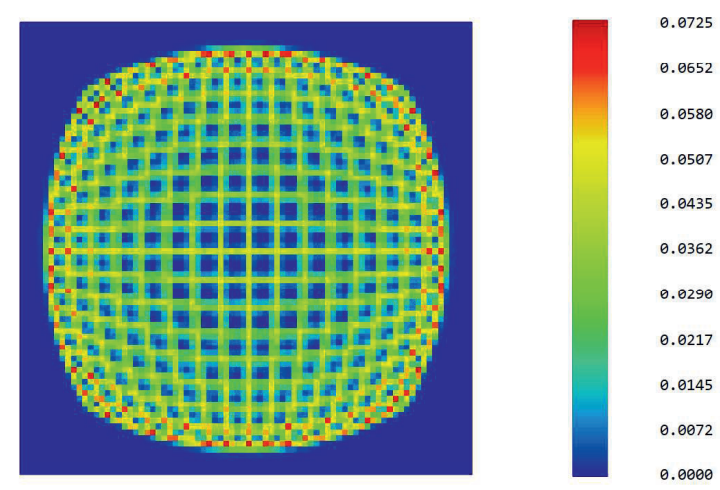

Fig. 2: Geometric Image analysis of an $80 \times 80$ pix grid to visualize the spatial resolution and spherical aberrations especially the distortion

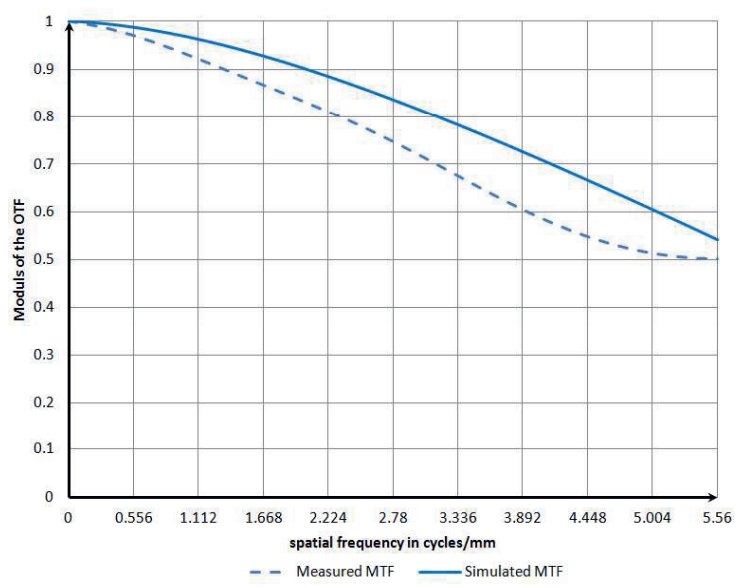

Fig. 3: FFT MTF Analysis simulated / measured for central pixels in a distance of $300 \mathrm{~mm}$
The developed optics is suitable for surveillance applications, where an area as large as possible has to be monitored. For the example of a $3 \mathrm{~m}$ room height it is possible to monitor an area of 6 meters (64 pixels) x 10.4 meters ( 80 pixels). In case of using the widest possible FOV of $150^{\circ}$ even a range of $22.4 \mathrm{~m}$ can be overviewed. Due to the low f-number and the small decrease of signal to the edge of the image, with a HTPA80x64d thermopile array it is possible to detect room temperature warm objects.

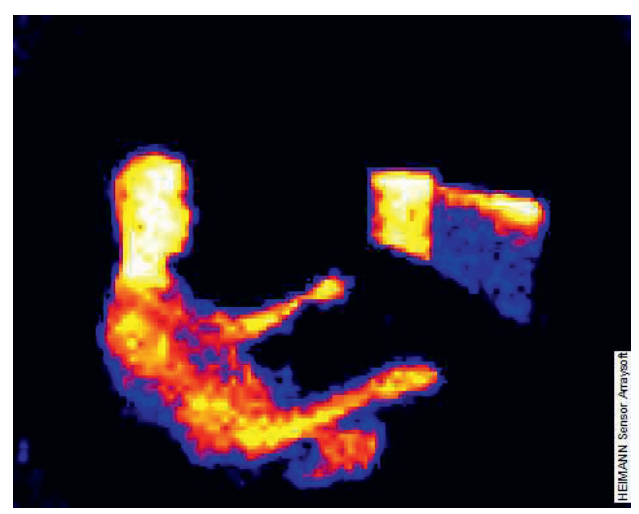

Fig. 4: Computer workstation with two screens in front of a person.

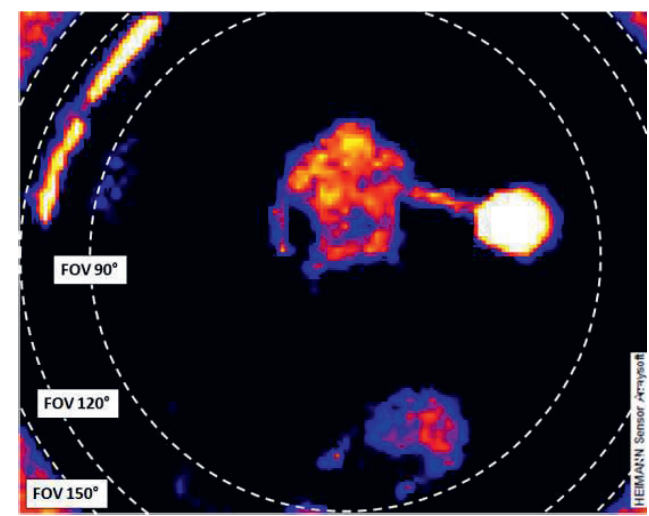

Fig. 5: Person carries a $40^{\circ} \mathrm{C}$ warm object through the surveillance area; thermopile array mounted at the $2.5 \mathrm{~m}$ high ceiling

There is an additional advantage of Thermopile arrays with > 5000 Pixels: They do not only allow to detect persons as hot spot. Together with a smart software, even a differentiation between humans and larger pets should be possible. 


\section{References}

[1] M. Schnorr, B. Forg, F. Herrmann, Dr. W. Leneke, Dr. M. Simon, Dr. J. Schieferdecker: "New miniaturized Thermopile IR Arrays with medium resolution", IRS ${ }^{2}$ Conference 2015, Proc., Nürnberg, Germany, pp. 957-960, DOI 10.5162/irs2015/4.4

[2] M. Schnorr, B. Forg, F. Hermann, Dr. W. Leneke, Dr. J. Schieferdecker, M. Schulze, Dr. M. Simon, K. Storck, L. Buydens „New Developments for Thermopile Array Sensors in Packages as small as TO 39" IRS ${ }^{2}$ Conference 2013, Proc., Nürnberg, Germany, pp. 28-31,

DOI 10.5162/irs2013/i2.2 